\title{
Manufacture of a fast neutron detector using EJ-301 liquid scintillator
}

\author{
Phan Van Chuan, Nguyen Duc Hoa, Nguyen Xuan Hai, Nguyen Duy Tan
}

\begin{abstract}
A fast neutron detector using the EJ301 scintillator was manufactured for study on detecting fast neutrons and gamma-rays. Detector characteristics include the energy linearity, the efficiency response and the neutron/gamma discrimination were guaranteed for neutron detection in the energy range from 50 to 3000 keVee. The ability discrimination of neutrons/gamma-rays of the detector was evaluated by the charge comparison (CC) method using an ${ }^{252} \mathrm{Cf}$ source. The total efficiencies when measured on ${ }^{22} \mathrm{Na},{ }^{137} \mathrm{Cs},{ }^{60} \mathrm{Co}$ and ${ }^{252} \mathrm{Cf}$ sources were obtained $17.8 \%, 3.9 \%, 9.8 \%$ and $14.8 \%$, respectively. The Figure of Merit (FoM) values of $\mathrm{CC}$ method were 0.4-1.55 for the range of energy 50-1000 keVee (keV electron equivalent).
\end{abstract}

Keywords_EJ-301 liquid scintillator, fast neutron detector, pulse shape discrimination

\section{INTRODUCTION}

$\mathrm{N}$ eutron detection is very important in research about the field of neutron, such as radiation safety, research material, scattering particles, particle physics, etc. The slow neutrons are commonly detected based on the nuclear reaction mechanism, while the fast-neutrons are detected based on elastic scattering mechanism with light nuclei such as hydrogenous, ${ }^{4} \mathrm{He}$ or organic scintillators [1,2]. Organic scintillator detectors are widely employed in studies with fast neutrons and gamma-rays by many good properties: the fast decay time, the relatively high light-output and a reasonably good efficiency for fast neutrons [1, 3]. EJ-301 organic scintillator was manufactured by ElJen Technology (or its equivalent, NE213, BC501A), the yield curve consists of two exponential decays - the fast and

Received: 13-9-2017; Accepted: 13-10-2017; Published: 30-8-2018

Phan Van Chuan ${ }^{1 *}$, Nguyen Duc Hoa ${ }^{1}$, Nguyen Xuan $\mathrm{Hai}^{2}$, Nguyen Duy Tan ${ }^{1}-{ }^{I}$ Dalat University; ${ }^{2}$ Dalat Nuclear Research Institute

*Email: chuanpv@dlu.edu.vn slow components of the scintillator light that depends on different kinds of radiation $[1,4,5]$. By coupling a photo multiplier tube (PMT) - to the scintillator, the light can be collected and converted into a voltage pulse, allowing for data acquisition/processing $[1,6]$. Those properties are commonly used to identify neutrons and gammarays by using pulse shape discrimination (PSD) techniques.

Many PSD algorithms have been evaluated and reported, such as zero-crossing (ZC) [6-8], PGA [9], CC [6-8, 9-11], frequency gradient analysis (FGA) [5], TCT [12], discrete Fourier transform (DFT) [13], CPR [14], etc. Among them, the CC and $\mathrm{ZC}$ algorithms are commonly implemented, therefore they have become the industrial standards which are used to compare with new discrimination algorithms $[5,6]$.

In the present study, a fast neutron detector was designed and manufactured using the EJ-301 liquid scintillator for neutron monitoring and training purposes. A preamplifier was also manufactured in order to make the suitable shaping pulse for data acquisition and processing. The qualities of the detector were assessed by the total efficiency, sensitivity and linearity with gamma-rays. The ability to distinguish between neutrons and gamma-rays was assessed through digital CC method. The CC method was implemented by a program in MATLAB software using the data that are digitized from the pulses of detector by a digital oscilloscope.

\section{MATERIALS AND METHODS}

\section{Detector manufacture}

The designed layout of the detector is shown in Fig.1, which consist of a liquid scintillator container (cell), a photo-multiplier tube (PMT), a voltage divider, a shield cover and a preamplifier. The cell is a right cylinder made of aluminum with $34 \mathrm{~mm}$ diameter $60 \mathrm{~mm}$ length in size. The 
inner surface of the cell was polished and matched PMT through ultra violet glass window with 2 mm thickness. The PMT Hamamatsu R9420 has $1.6 n s$ and $550 \mathrm{ps}$ rise time and transit time spread (FWHM), respectively [15]. The cell, PMT and preamplifier are housed inside the cover shield which is made of aluminum in the form of cylindrical, with $49 \mathrm{~mm}$ in diameter $200 \mathrm{~mm}$ in length. This cover prevents light from outside and magnetic interference. The high voltage, signal and power supply connectors are mounted at the tail of the detector.

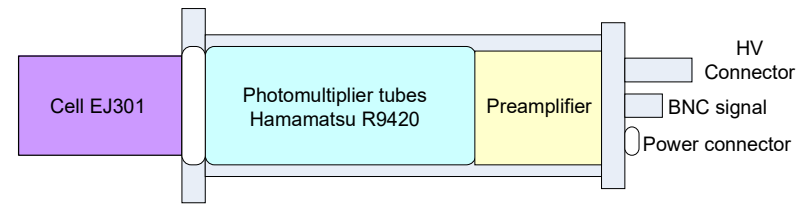

Fig. 1. Layout of neutron detector

The signals produced by the PMT have a very short rise time (less than $5 n s$ ) because the fast decays component of EJ-301 is 3.2 ns [4], so that the signal is distorted when it is transmitted to the digitized block, which is usually placed away from the detector [1]. The preamplifier consists of four main stages because the anode pulses produced by the PMT are current pulses, the first stage converts the current pulses to the voltage pulses using the load resistance $50 \mathrm{Ohms}$. The second stage amplifies the signal voltage from the first stage (gain of 30 times). The third stage is a filter using the second-order low-pass Sallenkey filter $\left(f_{-3 d B}=33.8 \mathrm{MHz}\right.$, Butterworth $\left.=0.6\right)$. The final stage has matched impedance to match cable impedance 50 Ohms. The Preamp would shape the pulses which had the rise time of approximately $12 \mathrm{~ns}$ and fall time of approximately $31 \mathrm{~ns}$ for the pulse of gammarays. The total amplifier voltage gain of the Preamp is $-17.85 \mathrm{~V} / \mathrm{V}$ and the output amplitude at the Compton edge of the ${ }^{137} \mathrm{Cs}$ source is $344.7 \mathrm{mV}$ and the ${ }^{60} \mathrm{Co}$ source is $806.8 \mathrm{mV}$, respectively. The total noise of preamplifier contribution to signal was $797.9 \pm 0.34 \mu \mathrm{V}$, which is equivalent to $1.13 \mathrm{keVee}$ calculated a calibration energy scale of the detector.

\section{Examined main characteristics of neutron detector}

The preamplifier was designed for linear output voltages in the 0 to $+2.2 \mathrm{~V}$ range, corresponds to range from 0 to $3100 \mathrm{keVee}$. A test setup is shown in Fig. 2 which the Preamplifier was tested in unconnected mode to PMT. The input of the Preamplifier was provided pulses from pulse generator (ORTEC Model 419), which was installed the rise time of $5 \mathrm{~ns}$ and fall time of 20 us. The amplitude and noise of both input and output pulses of the Preamplifier were measured by two channels of the digital Textronix Model DPO7254C (DPO7254C) that was installed in at 1 Giga samples per second (GSPS) and $2.5 \mathrm{GHz}$ bandwidth. For each input pulse amplitude, input/output amplitude values and the standard deviation $\sigma_{\text {In }} / \sigma_{\text {out }}$ of the pulses were measured by the DPO7254C. The amplitude of the input pulse was adjusted from 2.8 to $417 \mathrm{mV}$ by manual with 55 steps examined. The noise generated by preamplifier was calculated by the equation (1) [16].

$$
\sigma_{\mathrm{Pr} e}=\sqrt{\sigma_{\text {Out }}^{2}-\sigma_{\text {In }}^{2}}
$$

The results of the signal-to-noise ratio (SNR), the gains, sensitivity and linearity of preamplifier were shown in Table 1 and Fig.3.

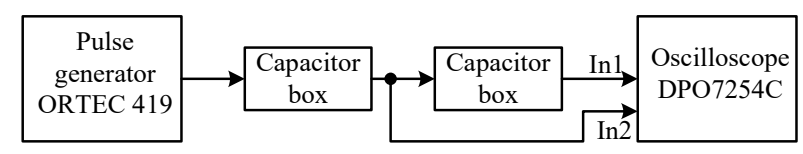

Fig. 2. The conguration of linearity, gain, noise and sensitivity evaluation for preamplifier

Table 1. The preamplifier parameters

\begin{tabular}{ll}
\hline Parameters & Values \\
\hline Measuring range & $0 \div 3000 \mathrm{keVee}$ \\
\hline Total noise & $797.9 \pm 0.34 \mu \mathrm{V}$ \\
\hline Baseline & $35.8 \pm 0.288 \mathrm{mV}$ \\
\hline Sensitivity & $707 \mathrm{mV} / \mathrm{MeV}$ \\
\hline
\end{tabular}

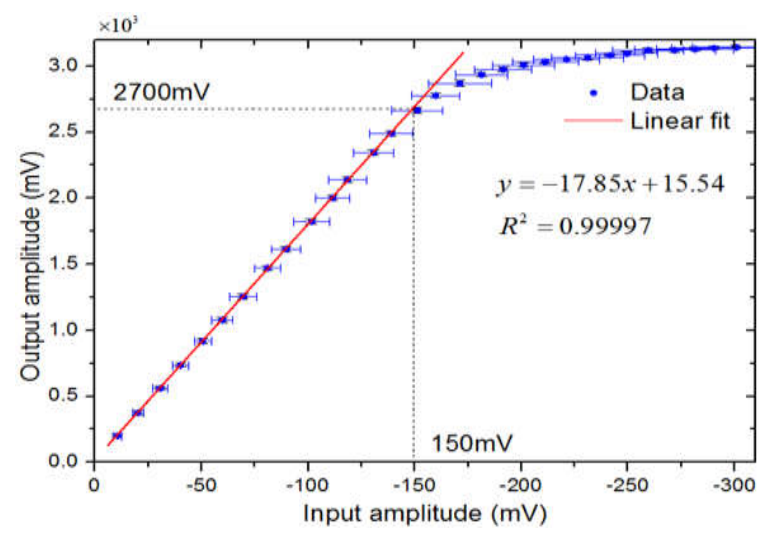

Fig. 3. The output versus input amplitude of preamplifier 
Because the light intensity of the EJ-301 liquid scintillator is good linearity on gamma sources $[1,4]$, this study uses three ${ }^{22} \mathrm{Na},{ }^{137} \mathrm{Cs}$ and ${ }^{60} \mathrm{Co}$ standard sources to evaluate the linearity of the detector. The relation the height of pulse with energy at the Compton edge of the gamma sources was used that evaluate the linearity of the detector with energy. The maximum backscatter energy $\left(E_{c}\right)$ was counted by equation (2) [1].

$$
E_{c}=E_{\gamma}\left(1-\frac{1}{1+\frac{2 E_{\gamma}}{m_{e} c^{2}}}\right)
$$

Where, $\mathrm{E}_{c}, \mathrm{E}_{\gamma}, m_{e}$ and $c$ are maximum backscatter energy, the energy of gamma-ray, electron rest mass, and speed of light in vacuum, respectively.

Table 2. Gamma energies from different nuclides corresponding to their calculated energies of Compton edge as a function of experimental channels measured by the MCA

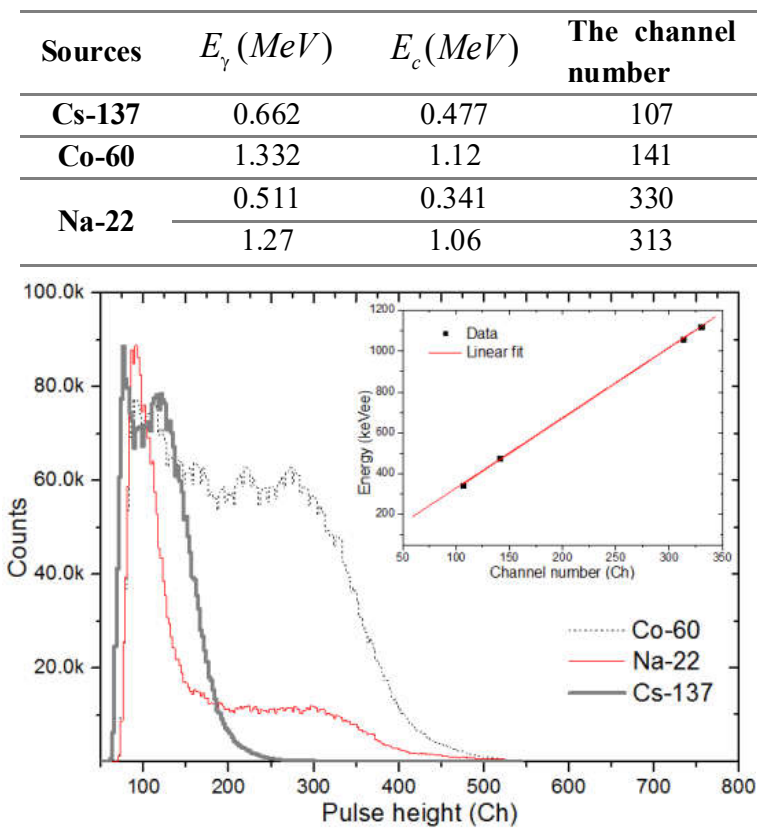

Fig. 4. Pulse height distribution from sources of ${ }^{60} \mathrm{Co},{ }^{22} \mathrm{Na}$ and ${ }^{137} \mathrm{Cs}$. The upper inset shows the calibration data using the Compton edges of the gamma-ray spectra

The Table 2 showed that measurements were performed with gamma-ray sources of ${ }^{22} \mathrm{Na}$, ${ }^{137} \mathrm{Cs}$ and ${ }^{60} \mathrm{Co}$, and each the measurement of those gamma sources were placed beside the monitor scintillation. Each the measurement of the pulse amplitude histogram was measured by the DPO7254C as the amplitude spectrum of the gamma source, respectively. The number of channels of the Compton edge corresponded to the $E_{c}$ of the gamma source, respectively. Because the Compton edge of the $1137.2 \mathrm{keV}$ peak of ${ }^{60} \mathrm{Co}$ was obscured by the that of $1332 \mathrm{keV}$ peak, only the Conton edge of the $1137.2 \mathrm{keV}$ peak was not used in the calibration. The energy spectra of ${ }^{60} \mathrm{Co},{ }^{22} \mathrm{Na}$ and ${ }^{137} \mathrm{Cs}$ sources are shown in Fig. 4, that used the oscilloscope DPO7254C which was operated in spectrum mode.



Fig. 5. Schematic view of assessing total efficiency and data acquisition system for EJ-301 detector

The total efficiency of the detector was evaluated by the schematic on Fig. 5. The total efficiency is defined as the ratio of the total number of events which are detected to the total number of gammaray incident on the detector. The total efficiencies of the detector were identified by ${ }^{22} \mathrm{Na}$ (activity on $12 / 2000$ was $9 \mu \mathrm{C}_{\mathrm{i}}$ ), ${ }^{137} \mathrm{Cs}$ (activity in $12 / 2001$ was $11 \mu \mathrm{C}_{\mathrm{i}}$ ), ${ }^{60} \mathrm{Co}$ (activity in $12 / 2000$ was $11 \mu \mathrm{C}_{\mathrm{i}}$ ), and ${ }^{252} \mathrm{Cf}$ (activity in $05 / 2011$ was $11.6 \mathrm{mC}_{\mathrm{i}}$ ) sources. The gamma sources are placed near the cell scintillator and placed $100 \mathrm{~cm}$ from the ${ }^{252} \mathrm{Cf}$ source to the detector (see Fig. 5). The pulses in these processes include gamma source, ${ }^{252} \mathrm{Cf}$ and background were counted by the Multi-ChannelAnalyzer (MCA) and spectrum analyzer software on a computer. The cross section of the liquid scintillator cell when decrease $5 \%$ by the air bubble was $19.4 \mathrm{~cm}^{2}$.

\section{Examined the ability of neutron-gamma discrimination}

In order to assess the ability to discriminate of the detector, this study used the ${ }^{252} \mathrm{Cf}$ source, which was placed at $100 \mathrm{~cm}$ from the detector (Fig. 5). The detector was biased high voltage of - 
$1200 \mathrm{~V}$ by the High Power Supply (Canberra 3002D); the detector's pulses were acquired by the DPO7254C which was set at 12 bit resolution, the bandwidth of $2.5 \mathrm{GHz}$ and at a sampling rate of 1 GSPS. The pulses were transferred to the PC for offline analysis by the PSD CC method. The program of PSD CC method was performed on MATLAB software and the results of the graph and FoMs were calculated by the Originlab 8.5 software.

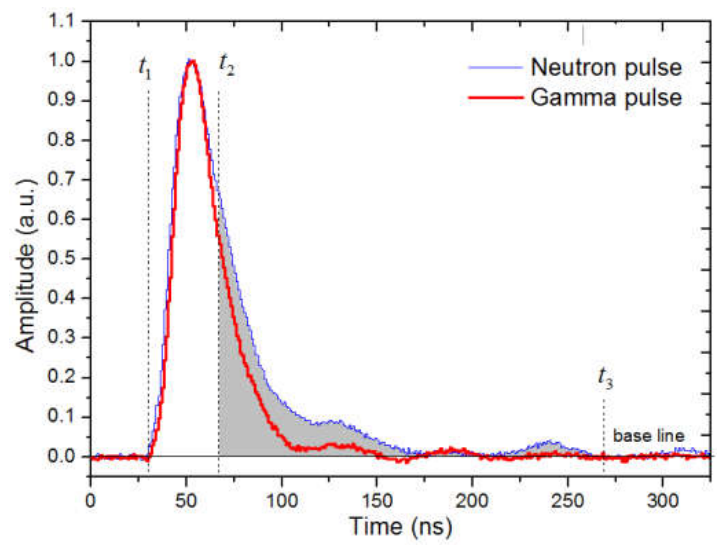

Fig. 6. Typical neutron and gamma-ray pulses in one sampling

The typical neutron and gamma - ray pulses with the same amplitude of the EJ-301 detector were shown in Fig. 6. The neutron pulses exhibited a larger decay time to the baseline, so with the same amplitude neutron/gamma pulses the area of the tail of the neutron pulse was greater than that of the gamma pulse. The digital PSD method chosen for comparison consists of integration techniques were applied to digitized

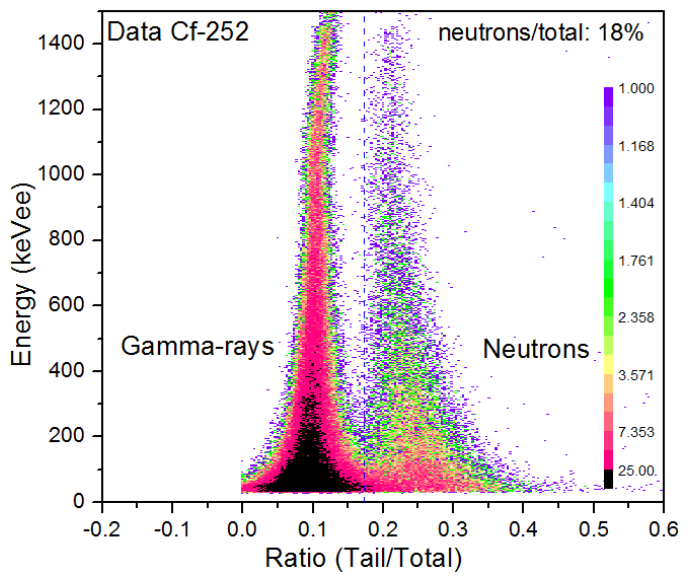

(A) pulses, where each pulse was integrated twice, using two different ranges [7-10, 14]. The total integral was calculated for full pulse that began is at the start point $\left(t_{l}\right)$ to an optimal point at the tail pulse $\left(t_{3}\right)$. The tail integral was calculated in range begins at a fixed position after the pulse maximum $\left(t_{2}\right)$ and also extended to the last data point chosen in the total integral range $\left(t_{3}\right)$. The survey data indicate that the separation was the best where $t_{2}$ was $20 n s$ and $t_{3}$ was $210 n s$ after the pulse maximum. The PSD parameters could be created using the ratio values between the tail and total integrals. The PSD parameter of neutron pulses was larger than that of gamma pulses.

\section{RESULTS AND DISCUSSION}

The measured data with a neutron source ${ }^{252} \mathrm{Cf}$ and ${ }^{60} \mathrm{Co}$ were analyzed by the PSD CC method. The scatter plots of the neutron-gamma separation with an energy threshold of $50 \mathrm{keVee}$ by the CC method are shown in Fig. 7 (a) and (b), respectively. In the region of the energy survey shown that the threshold over $200 \mathrm{keVee}$ the ability to distinguish between neutrons and gamma-rays very well. While below the 200keVee threshold the ability to distinguish between neutrons and gamma-rays was not good and at the threshold $50 \mathrm{keVee}$ the discrimination was not clear for neutron and gamma. The statistical chart of the $\mathrm{CC}$ method at energy threshold $300 \mathrm{keVee}$ was shown that the ability to distinguish between neutrons and gamma-rays was very clear $(\mathrm{FoM}=1.22)$.



(B)

Fig. 7. The scatter plot of charge comparison: (A) the scatter plot of ${ }^{252} \mathrm{Cf}$, (B) the scatter plot of ${ }^{60} \mathrm{Co}$ 


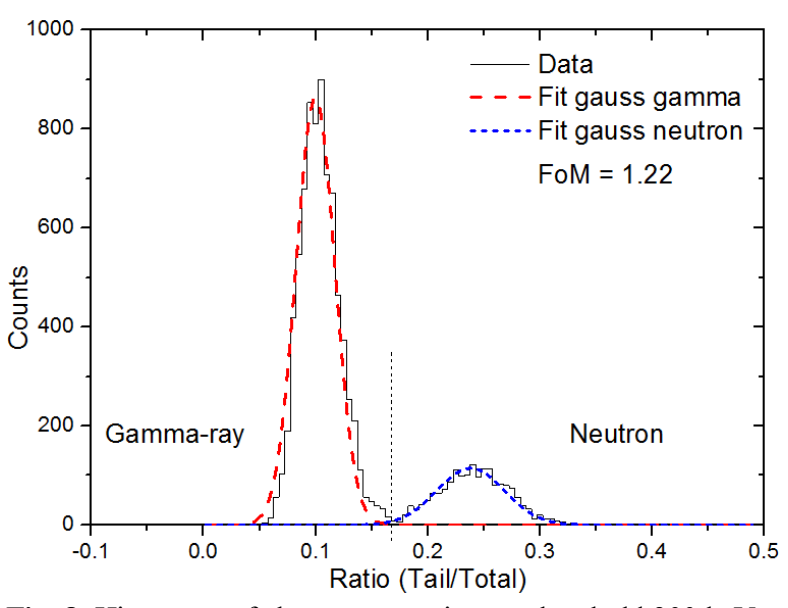

Fig. 8. Histogram of charge comparison at threshold $300 \mathrm{keVee}$

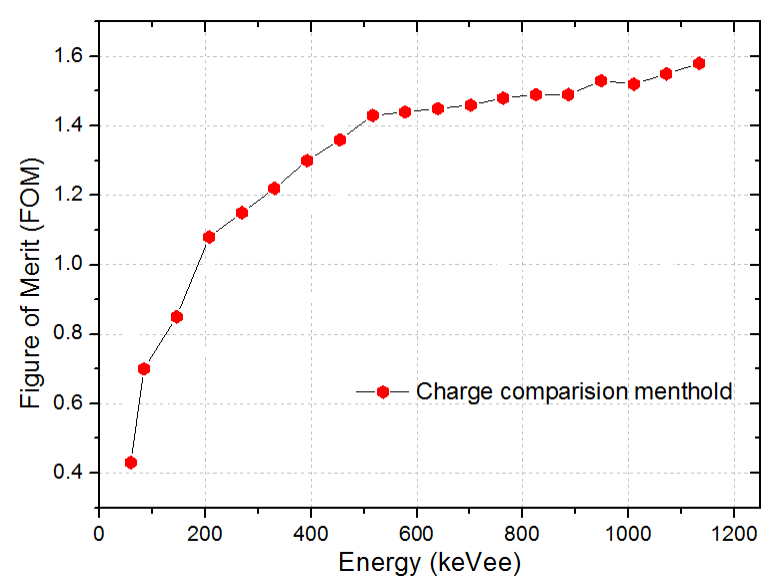

Fig. 9. The FoM values as a function of energy threshold corresponding of $\mathrm{CC}$ method in the range of energy from 50 to $1100 \mathrm{keVee}$

Fig. 9 showed the FoM values as a function of threshold in a range of energy from 50 to $1100 \mathrm{keVee}$. The FoMs were approximately 0.43 at $50 \mathrm{keVee}$ and greater than 1.0 at $200 \mathrm{keVee}$ energy threshold. At the $83 \mathrm{keVee}$ energy threshold, the FoM was measured 0.7 and its reached the value 1.15 at the $200 \mathrm{keVee}$ energy threshold. At the $1000 \mathrm{keVee}$ energy threshold, the FoM increased of 1.55. These results were similar as the presented in Ref. [7, 8, 11].

Table 3. The total efficiency value determined by ${ }^{252} \mathrm{Cf}$, ${ }^{137} \mathrm{Cs},{ }^{22} \mathrm{Na}$ and ${ }^{60} \mathrm{Co}$ sources

\begin{tabular}{cccc}
\hline Sources & $\begin{array}{c}\text { Activity } \\
(\mathbf{B q})\end{array}$ & $\begin{array}{c}\text { Count } \\
\text { rate (cps) }\end{array}$ & $\begin{array}{c}\text { Total } \\
\text { efficiency } \\
\mathbf{( \% )}\end{array}$ \\
\hline${ }^{252} \mathbf{C f}$ & $1,052 \times 10^{7}$ & 88,906 & 14.8 \\
\hline${ }^{60} \mathbf{C o}$ & 47,962 & 1,732 & 9.8 \\
\hline${ }^{137} \mathbf{C s}$ & 94,474 & 3,869 & 3.9 \\
\hline${ }^{22} \mathbf{N a}$ & 4,397 & 440 & 17.8 \\
\hline Background & \multicolumn{3}{c}{182} \\
\hline \multicolumn{2}{c}{ Note: * neutron source was closed }
\end{tabular}

The results of the total efficiency of the detector were surveyed by ${ }^{22} \mathrm{Na},{ }^{137} \mathrm{Cs},{ }^{60} \mathrm{Co}$ and ${ }^{252} \mathrm{Cf}$ sources (Table 3 ). The survey values showed that the total efficiency was maximum for the ${ }^{22} \mathrm{Na}$ source. The events of both 511 and $1274.5 \mathrm{keV}$ peaks were used for canculated total efficiency. The total efficiency on the ${ }^{252} \mathrm{Cf}$ reached $14.8 \%$ that was measured with both neutron and gamma events. Determining exactly the efficiency of the EJ-301 was quite complex by the inadequate standard sources and the bad resolution of the EJ301 liquid scintillator. This issue is still being studied by the authors and will be published in another time.

\section{CONCLUSION}

A scintillation detector using the EJ-301 liquid scintillator has been designed and built for fastneutron measurements. The detector is designed to measure in the 50 to $3000 \mathrm{keVee}$ energy range corresponding to an output voltage of $35.8 \mathrm{mV}$ to $2200 \mathrm{mV}$, which was compatible with the input voltage range of the high speed ADCs that it could directly interconnect. The sensitivity of the detector was $707 \mathrm{mV} / \mathrm{MeV}$. The most important characteristic of the neutron detector was the ability to discriminate between neutrons and gamma-rays to eliminate gamma-rays noise in fast-neutron measurements that have been evaluated by the PSD CC method. Those results showed that the EJ-301 detector could be used in system fast-neutron measurements by digital technology.

\section{REFERENCES}

[1] G.F. Knoll, Radiation Detection and Measurement, John Wiley \& Sons (2010).

[2] R. Aryaeinejad, E.L. Reber, D.F. Spencer, "Development of a Handheld Device For Simultaneous Monitoring of Fast Neutrons and Gamma Rays", IEEE Trans. Nucl. Sci., vol. 49, no. 4, pp. 1909, 2002.

[3] S.D. Jastaniah, P.J. Sellin, "Digital pulse-shape algorithms for scintillation-based neutron detectors", IEEE Trans. Nucl. Sci., vol. 49, no. 4, pp. 1824-1828, 2002.

[4] EJ-301, EJ-309 datasheet, Eljen Technology, 2016.

[5] G. Liu, M.J. Joyce, X. Ma, M.D. Aspinall, “A digital method for the discrimination of neutrons and rays with organic scintillation detectors using frequency gradient analysis”, IEEE Trans. Nucl. Sci., vol. 57, pp. 1682-1691, 2010.

[6] C.S. Sosa, M. Flaska, S.A. Pozzi, "Comparison of analog 
and digital pulse-shape-discrimination systems", Nucl. Inst. And Meth. A, 826, 72-79, 2016.

[7] W. Bo, Z.X. Ying, C. Liang, G.E. Hong-Lin, M.A. Fei, Z. Hong-Bin, J.U. Yong-Qin, Z. Yan-Bin, L. Yan-Yan, X.U. Xiao-Wei, "Study of digital pulse shape discrimination method for $n-\gamma$ separation of EJ-301 liquid scintillation detector", Chinese Physics C, vol. 37, no. 1, 010201, 2013.

[8] M. Nakhostin, P.M. Walker, "Application of digital zerocrossing technique for neutron-gamma discrimination in liquid organic scintillation detectors", Nucl. Inst. and Meth. $A$, vol. 621, 498501, 2010.

[9] B.D. Mellow, M.D. Aspinall, R.O. Mackin, M.J. Joyce, A.J. Peyton, "Digital discrimination of neutrons and $\gamma$-rays in liquid scintillators using pulse gradient analysis", Nucl. Inst. and Meth. A, vol. 578, 191-197, 2007.

[10] M.L. Roush, M.A. Wilson, W.F. Hornyak, "Pulse shape discrimination", Nucl. Inst. And Meth. A, vol. 31, 112-124, 1964.

[11] C. Payne, P.J. Sellin, M. Ellis, K. Duroe, A. Jones, M. Joyce, G. Randall, R. Speller, "Neutron/gamma pulse shape discrimination in EJ-299-34 at high flux", IEEE Nuclear Science Symposium and Medical Imaging Conference
(NSS/MIC), 2015.

[12] M. Amiri, V. Prenosil, F. Cvachovec, Z. Matej, F. Mravec, J. Radioanal, "Quick algorithms for real-time discrimination of neutrons and gamma rays", Nucl. Chem., vol. 303, pp. 583-599, 2015.

[13] M.J. Safari, F.A. Davani, H. Afarideh, S. Jamili, E. Bayat, "Discrete Fourier Transform Method for Discrimination of Digital Scintillation Pulses in Mixed Neutron-Gamma Fields", IEEE Trans. Nucl. Sci., vol. 63, no. 1, pp. 325-332, 2016.

[14] D. Takaku, T. Oishi, M. Baba, "Development of neutron-gamma discrimination technique using patternrecognition method with digital signal processing", Prog. Nucl. Sci. Technol., vol. 1, pp. 210-213, 2011.

[15] R9420 Datasheet, Hamamatsu, 2014.

[16] IEEE Std 301-1988, The Institute of Electrical and Electronics Engineers, Inc, (1989).

\title{
Chế tạo đầu đo neutron nhanh sử dụng nhấp nháy lỏng EJ-301
}

\author{
Phan Văn Chuân ${ }^{1, *}$, Nguyễn Đức Hòa ${ }^{1}$, Nguyễn Xuân Hải ${ }^{2}$, Nguyễn Duy Tân ${ }^{1}$ \\ ${ }^{1}$ Trường Đại học Đà Lạt, ${ }^{2}$ Viện nghiên cứu hạt nhân Đà Lạt \\ *Tác giả liên hệ: chuanpv@dlu.edu.vn \\ Ngày nhận bản thảo: 13-09-2017; Ngày chấp nhận đăng: 13-10-2017; Ngày đăng: 30-8-2018
}

Tóm tắt-Một đetectơ nơtron nhanh sử dụng nhấp nháy EJ-301 đã được chế tạo phục vụ cho nghiên cứu nơtron nhanh và tia gamma. Các thuộc tính chính của detector bao gồm độ tuyến tính năng lượng, hiệu suất ghi và khả năng phân biệt nơtron gamma đã được kiểm tra trong vùng năng lượng khảo sát từ $50 \div 3000 \mathrm{keVee}(\mathrm{keV}$ tương đương). Khả năng phân biệt nơtron - gamma của đetectơ được đánh giá thông qua phương pháp so sánh diện tích xung sử dụng nguồn ${ }^{252} \mathrm{Cf}$. Các hiệu suất tổng đo được trên các nguồn ${ }^{22} \mathrm{Na},{ }^{137} \mathrm{Cs},{ }^{60} \mathrm{Co}$ và ${ }^{252} \mathrm{Cf}$ đạt các giá trị tương ứng $17,8 \%, 3,9 \%, 9,8 \%$ và $14,8 \%$. Hệ số phẩm chất (Figure of Merit: FoM) đánh giá cho phương pháp so sánh diện tích xung của đetectơ đạt $0,4 \div 1,55$ trong vùng năng lượng khảo sát (50 $\div 1000$ keVee).

Tù̀ khóa-đetectơ nơtron nhanh, nhấp nháy lỏng EJ-301, phân biệt dạng xung 